\title{
Reducción del tiempo y de la temperatura de sellado del aluminio anodizado con aditivos orgánicos
}

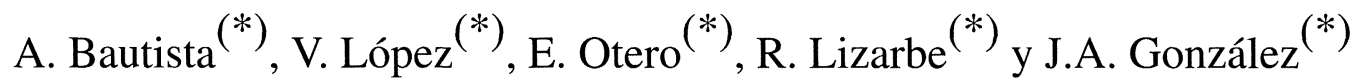

Resumen Diferentes procesos de sellado de las capas anódicas de óxido de aluminio se han utilizado industrialmente desde hace más de 30 años. En los dos decenios precedentes se ha realizado un gran esfuerzo para reducir los costes de la operación del sellado hidrotérmico tradicional, en agua desionizada a ebullición (SHT), una operación muy costosa por su duración y la temperatura a la que se desarrolla. Se proponen nuevos procedimientos de sellado en los que, mediante la utilización de aditivos orgánicos, se reduce sustancialmente el tiempo del SHT o la temperatura del mismo.

Palabras clave: Anodizado. Nuevos métodos de sellado. Reducción del tiempo. Reducción de la temperatura.

\section{Time and temperature reduction of the sealing process of porous aluminium oxide films with organic additives}

\begin{abstract}
Different sealing processes of anode coatings in aluminium oxide have been industrially used for more than 30 years. In two of the preceeding decades a great effort was realized to reduce costs in the traditional hydrothermal sealing process in deionized boiling water (SHT), a very expensive process due to its endurance and high temperature on which it develops. New sealing procedures are proposed, on which by means of the use of organic additives, the time or the temperature of the SHT is essentially reduced.
\end{abstract}

Keywords: Anodizing. New sealing methods. Sealing times reduction. Lowering sealing tempertures.

\section{INTRODUCCIÓN}

La mayor parte del aluminio anodizado empleado en arquitectura se anodiza en baños de ácido sulfúrico, obteniéndose un recubrimiento poroso con una elevada capacidad absorbente. Estas características hacen que los anodizados sean susceptibles al ensuciamiento y a la corrosión en medios agresivos, requiriendo, en muchas aplicaciones, un procedimiento de sellado de los poros. En la industria, el sellado se ha hecho tradicionalmente por inmersión en agua desionizada a ebullición, procedimiento conocido como sellado hidrotérmico (SHT).

Debido a la temperatura a la que se realiza y a su larga duración, $>2 \mathrm{~min} / \mu \mathrm{m}$ (1 y 2), el SHT resulta

(*) Centro Nacional de Investigaciones Metalúrgicas, CENIM (CSIC), Avda. de Gregorio del Amo, 8. 28040-Madrid (España). una operación costosa. Esto explica que, desde antiguo, se haya tratado de aminorar estos problemas con adiciones al baño de sellado, siendo las adiciones de sales de níquel, de acetatos y de ciertas aminas, las más empleadas (3-5). Incluso, desde comienzos del decenio de los 80 , se han ido introduciendo procedimientos alternativos de sellado en frío, que trabajan a temperatura ambiente, basados en soluciones de fluoruro de níquel (2 y 6 ).

Sin embargo, las mismas características absorbentes de este tipo de anodizados multiplican enormemente sus aplicaciones en arquitectura, a causa de su facultad para adoptar las más diversas coloraciones. La mayor dureza del óxido, con respecto al metal, protege a éste contra la abrasión, corrigiendo uno de sus escasos defectos. En atmósferas poco agresivas, la capacidad absorbente de los anodizados sin sellar promueve un proceso de autosellado, que prevalece sobre el deterioro y termina por 
garantizar una calidad que supera ampliamente las exigencias de la normativa vigente (7 y 8).

El propósito de esta investigación es proponer nuevos procedimientos de sellado en los que, mediante la utilización de aditivos orgánicos, se reduce sustancialmente el tiempo de sellado a la temperatura de ebullición, o bien la temperatura de la operación.

\section{MATERIALES Y METODOS EXPERIMENTALES}

\subsection{Materiales}

Como electrodos de trabajo se utilizaron probetas de aluminio de pureza comercial $(99,5 \%)$, de $5 \times 10 \mathrm{~cm}$, anodizadas bajo condiciones técnicas en solución de ácido sulfúrico $(18 \%)$, a $20{ }^{\circ} \mathrm{C}$ y 1,5 $\mathrm{A} / \mathrm{dm}^{2}$. El espesor de las capas de óxido fue, habitualmente, de $20 \pm 1 \mu \mathrm{m}$ y se sometieron a diferentes tiempos de sellado en agua desionizada, con y sin aditivos orgánicos, a temperaturas comprendidas entre $50{ }^{\circ} \mathrm{C}$ y la de ebullición del agua. También se estudió el efecto combinado de sellados incompletos con envejecimientos, en atmósferas de elevada humedad relativa, a temperatura ambiente y a $50^{\circ} \mathrm{C}$.

\subsection{Métodos de ensayo}

Para las distintas condiciones de sellado se realizaron los ensayos normalizados de disolución ácida en mezcla fosfocrómica, de admitancia y de la gota de colorante, comparándolos con medidas de impedancia llevadas a cabo en solución de $\mathrm{K}_{2} \mathrm{SO}_{4}$ del $3 \%$ en masa, aireada, sin agitación y a $25 \pm 1{ }^{\circ} \mathrm{C}$. El campo de frecuencias utilizado fue el comprendido entre $100 \mathrm{kHz}$ y $1 \mathrm{mHz}$. También se determinó la microestructura de las capas anódicas, con el microscopio electrónico de transmisión, para las distintas calidades de sellado.

\section{RESULTADOS Y DISCUSION}

Las propiedades de los anodizados dependen fundamentalmente de su carácter poroso, puesto de manifiesto por observación con el microscopio electrónico de transmisión (Fig. 1), que permite conocer su microestructura e incluso la subestructura de la pared de las celdas y del relleno de los poros (Fig. 2). Se puede esclarecer así el efecto de cualquier aditivo del baño de sellado o factor de corrosión sobre el mecanismo y la cinética de los procesos de sellado y envejecimiento (durabilidad) de las capas anódicas. Mediante cálculo, a partir de las dimensiones de poro y celdilla hexagonal dedu-

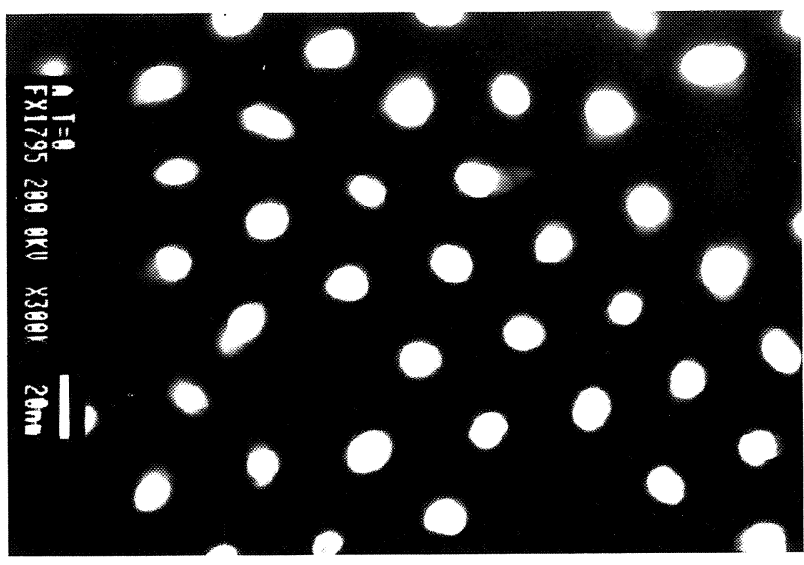

FIG. 1.- Sección transversal de las capas anódicas porosas, obtenidas en ácido sulfúrico, observadas con el microscopio electrónico de transmisión.

FIG. 1.- TEM micrograph of the cross section of porous anodic layers obtained in sulphuric acid.

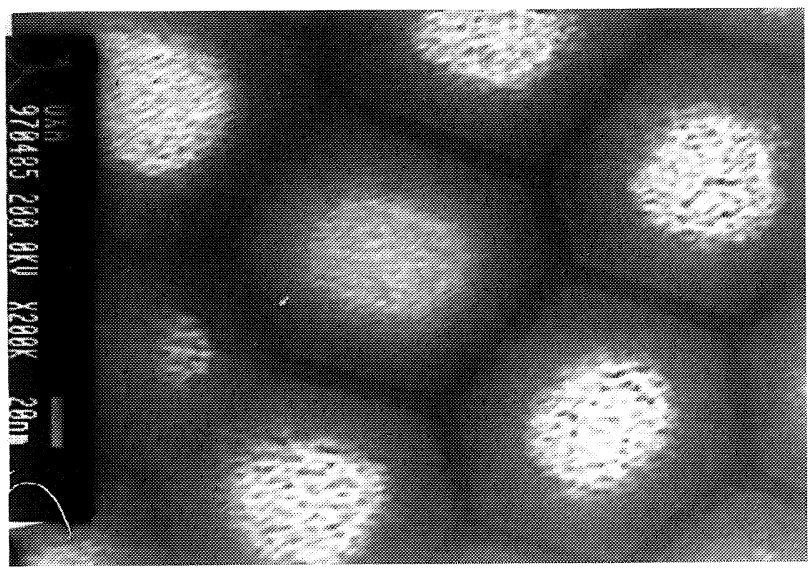

FIG. 2.- Sección transversal de las capas anódicas porosas, obtenidas en ácido oxálico, observadas con el microscopio electrónico de transmisión.

FIG. 2.- TEM micrograph of the cross section of porous anodic layers obtained in oxalic acid.

cibles de la figura 1 , se llega a una superficie específica de $18 \mathrm{~m}^{2} / \mathrm{g}$, y a un volumen de poros de $0,063 \mathrm{~cm}^{3} / \mathrm{g}$, datos prácticamente coincidentes a los obtenidos con porosimetría por absorción de nitrógeno (9).

En el SHT, en agua desionizada a ebullición, se ha conseguido, mediante aditivos orgánicos, esencialmente acetatos, alcoholes y aminas, reducir el tiempo de sellado necesario para cumplir la normativa vigente por un factor de 5 ó superior (Fig. 3). Recuérdese, al respecto, que la huella residual en la gota de colorante debe ser inferior a 2, menor de 30 $\mathrm{mg} / \mathrm{dm}^{2}$ la disminución de masa en el ensayo de disolución ácida en mezcla fosfocrómica y de $20 \mu \mathrm{S}$ la admitancia a $1 \mathrm{kHz}(10)$. Esto permite un considerable ahorro de energía (tiempo) y espacio, 


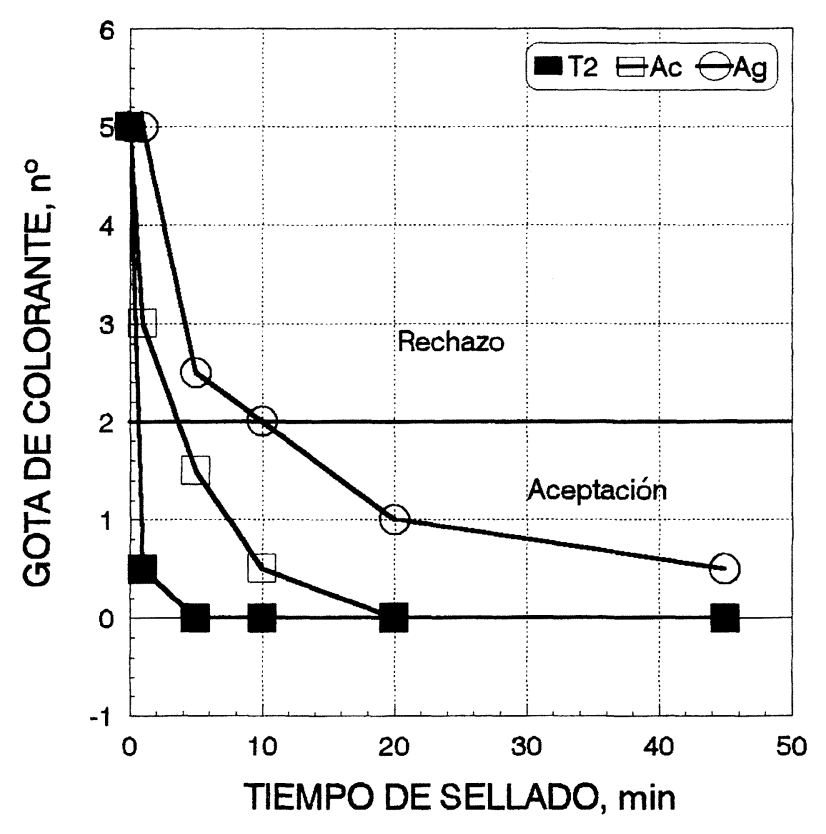

FIG. 3.- Evolución de la gota de colorante, con el tiempo de sellado a la temperatura de ebullición, en agua desionizada sin aditivos y con $0,5 \mathrm{~g} / \mathrm{L}$ de anión acetato y $2 \mathrm{cc} / \mathrm{L}$ de $\mathrm{T} 2$.

FIG. 3.-Evolution of the results of the dye drop test with the sealing time at boiling temperature, in de-ionized water without additives and with $0.5 \mathrm{~g} / \mathrm{L}$ of acetate anion and $2 \mathrm{cc} / \mathrm{L}$ of $T 2$.

pues con un solo baño de sellado se podría procesar la producción de 2 ó más baños de anodización. El efecto catalizador de los aditivos orgánicos empleados también es notable a temperaturas inferiores a la de ebullición del agua, como testimonia la figura 4, para el caso del ensayo de admitancia, en baños de sellado mantenidos a $50^{\circ} \mathrm{C}$.

De figuras como la 3 y la 4 se pueden deducir los tiempos críticos para superar las exigencias de la normativa vigente que, como demuestran los resultados de la figura 5 , crecen exponencialmente al disminuir la temperatura de sellado. El efecto de los aditivos es relativamente mayor cuanto menor sea la temperatura, y si a $100{ }^{\circ} \mathrm{C}$ se reducen los tiempos críticos de sellado por un factor de 5, a 50 ${ }^{\circ} \mathrm{C}$ se logra un factor próximo a 100 (Fig. 5). Para iguales tiempos de sellado, se ha conseguido obtener, con aditivos orgánicos, las mismas calidades a $80^{\circ} \mathrm{C}$ que a la temperatura de ebullición en su ausencia (Fig. 5). Esto supone un ahorro muy significativo de energía y una sustancial reducción de los problemas de condensaciones en las plantas.

No obstante, la reducción de la cinética de sellado al disminuir la temperatura (Figs. 4 y 5) impide alcanzar las calidades exigidas en tiempos compatibles con la práctica industrial, necesitándose, adicionalmente, un breve período de envejecimiento.

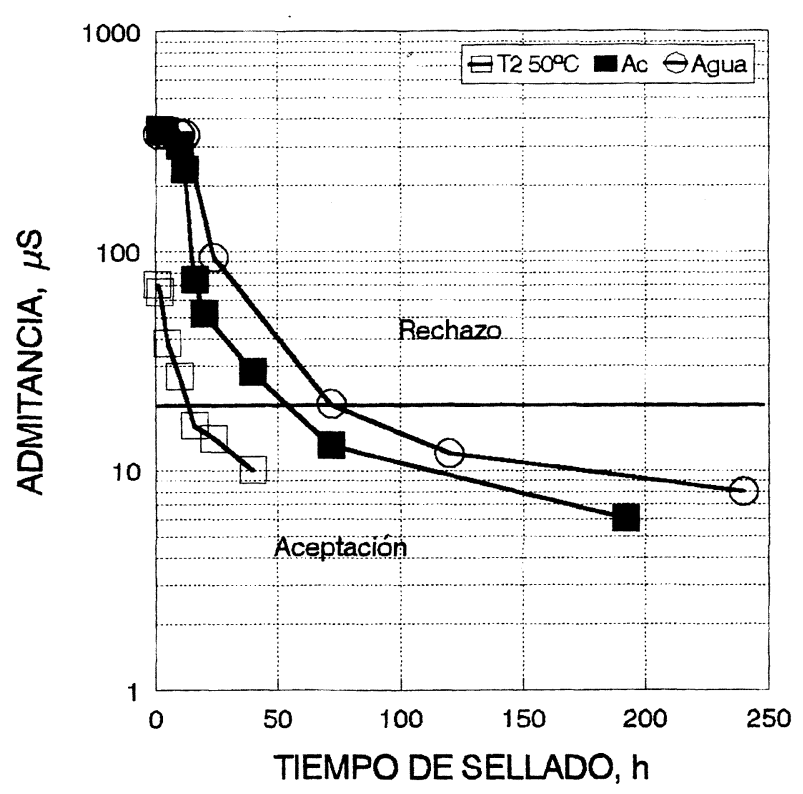

FIG. 4.- Evolución de la admitancia a $1 \mathrm{kHz}$, con el tiempo de sellado a $50{ }^{\circ} \mathrm{C}$, en agua desionizada sin aditivos y con $0,5 \mathrm{~g} / \mathrm{L}$ de anión acetato y $2 \mathrm{cc} / \mathrm{L}$ de $\mathrm{T} 2$.

FIG. 4.- Evolution of the admittance at $1 \mathrm{kHz}$ with the sealing time at $50{ }^{\circ} \mathrm{C}$, in de-ionized water without additives and with $0.5 \mathrm{~g} / \mathrm{L}$ of acetate anion and $2 \mathrm{cc} / \mathrm{L}$ of $T 2$.

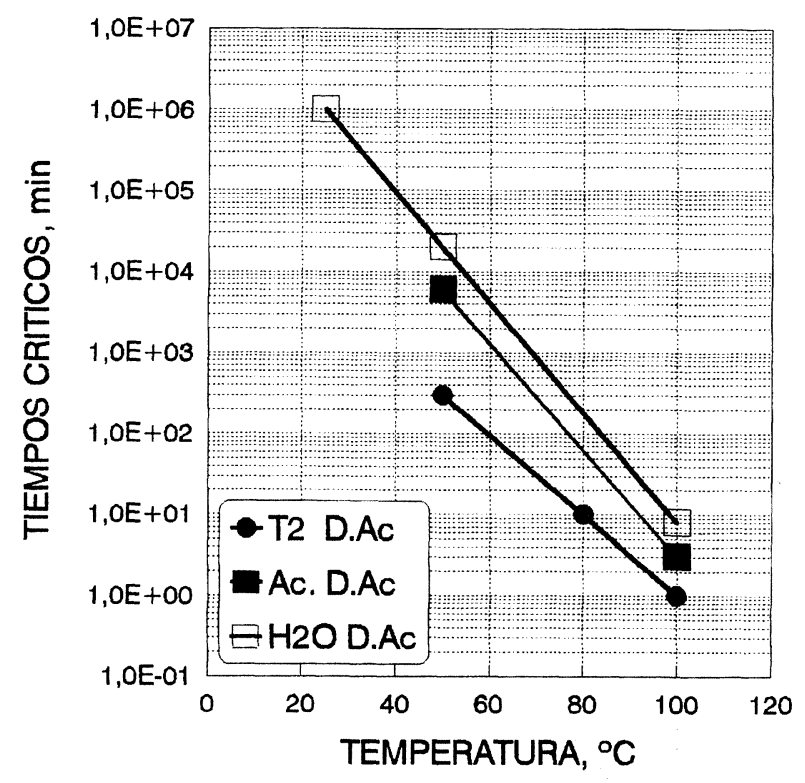

FIG. 5.- Tiempos mínimos requeridos para superar el ensayo de disolución ácida, en función de la temperatura y de la composición del baño de sellado.

FIG. 5.-Minimum times required to pass the acid dissolution test, as a function of the temperature and composition of the sealing bath.

El aumento de la calidad de los sellados incompletos, durante su exposición a la atmósfera, parece ser 
una característica universal de los mismos, siempre que se supere un mínimo de humedad relativa no establecido hasta el momento, si bien dicha mejora es mucho más rápida en cámara húmeda (Fig. 6) que en una atmósfera con precipitaciones escasas, como la de Madrid (Fig. 7).

El período de envejecimiento para satisfacer los mínimos de calidad estipulados en la normativa depende, naturalmente, de la temperatura, siendo inferior a 2 semanas si se efectúa a temperatura ambiente, y tan sólo de $24 \mathrm{~h}$ si se eleva la temperatura de envejecimiento a $50{ }^{\circ} \mathrm{C}$, siempre que la humedad relativa sea elevada (Fig. 6).

Los sellados incompletos aceleran considerablemente los procesos de autosellado-envejecimiento, como prueban las figuras 6 y 7 , asegurando la consecución de los mínimos de calidad exigidos industrialmente con períodos breves de exposición a la atmósfera. Además, los sellados insuficientes reducen sustancialmente la capacidad absorbente de los anodizados sin sellar, de manera que se conserva el aspecto casi tan eficazmente como en los sellados que superan inicialmente las exigencias de las normas.

Este carácter "catalítico" de los sellados incompletos sobre el envejecimiento, convierte en muy

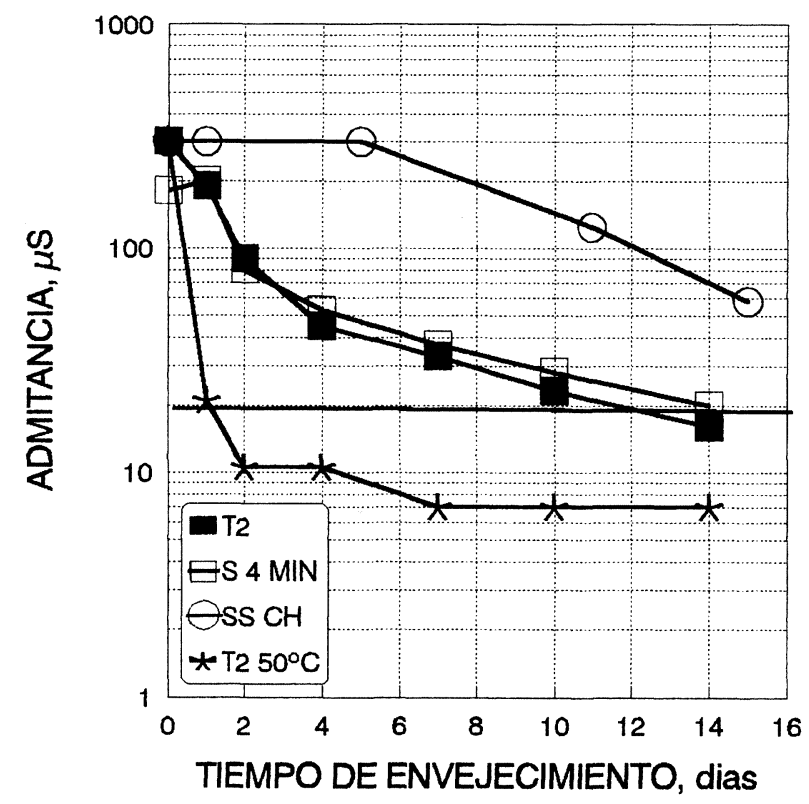

Fig. 6.- Evolución de la admitancia durante el envejecimiento en cámara húmeda, a temperatura ambiente, de probetas anodizadas sin sellar, selladas 4 min en agua a ebullición y 30 min en T2 a 50 ${ }^{\circ} \mathrm{C}$. Estas últimas se envejecieron también a $50^{\circ} \mathrm{C}$.

FIG. 6. - Variation of the admittance during the aging in wet chamber, at room temperature, of anodized specimens, unsealed, sealed $4 \mathrm{~min}$ in boiling water and $30 \mathrm{~min}$ in $\mathrm{T} 2$ at $50^{\circ} \mathrm{C}$. The last ones were also aged at $50^{\circ} \mathrm{C}$.

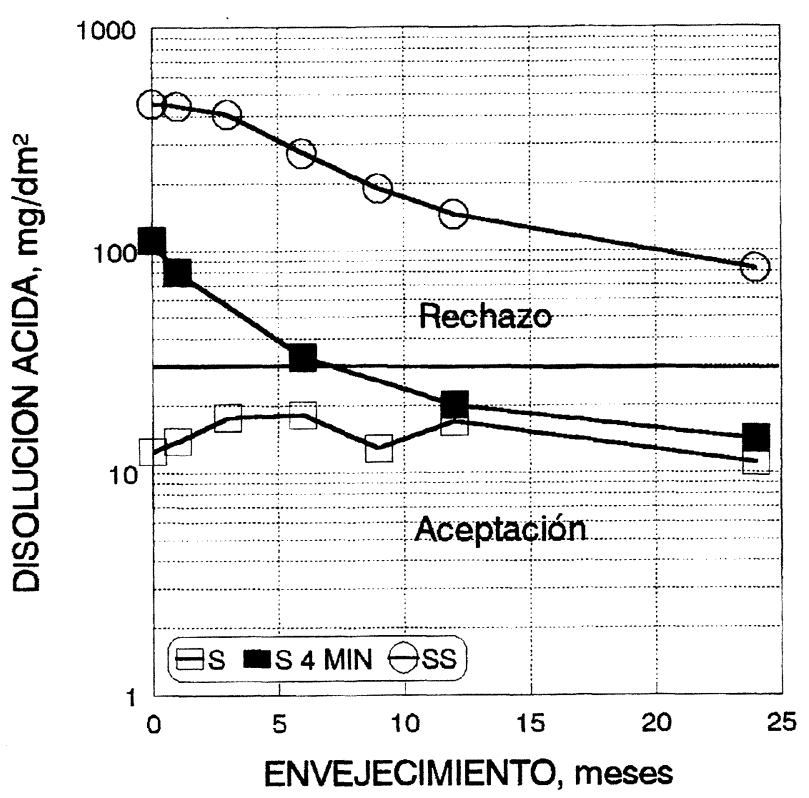

FIG. 7.- Evolución en la disolución ácida durante la exposición atmosférica de probetas anodizadas sin sellar, selladas 4 min en agua a ebullición y 30 min en $\mathrm{T} 2$ a $50^{\circ} \mathrm{C}$.

FIG. 7.- Evolution of the results of the acid dissolution test during the atmospheric exposure of anodized specimens, unsealed, sealed $4 \mathrm{~min}$ in boiling water and $30 \mathrm{~min}$ in $\mathrm{T} 2$ at $50^{\circ} \mathrm{C}$.

atractiva la combinación de un presellado a temperatura moderada, por ejemplo a $50{ }^{\circ} \mathrm{C}$, en un baño con aditivos orgánicos, con un breve período de envejecimiento. Los aditivos orgánicos, aunque necesitan una temperatura de impregnación algo superior a la de los sellados en frío y un período de envejecimiento algo más largo, tienen las ventajas de necesitar un control menos engorroso del baño de sellado y de prescindir de las sales de níquel, costosas y que pueden producir reacciones alérgicas.

La posibilidad de discernimiento de los ensayos normalizados se agota para calidades de sellado que superen ligeramente las exigidas en las normas, como prueban las figuras 3 y 7 para los ensayos de la gota de colorante y el de la disolución ácida, respectivamente. En cambio, los ensayos de impedancia, a partir de los cuales se pueden estimar las características físico-químicas de las capas porosa y barrera, muestran una sensibilidad mucho mayor, casi ilimitada (Fig. 8), ofreciendo una herramienta idónea para obtener una información detallada sobre el efecto de cualquier factor sobre la calidad y propiedades de las capas anódicas de óxido de aluminio. Se puede demostrar así que estas siguen evolucionando durante meses, años y decenios después de la operación de sellado (Fig. 8) (7 y 8). 


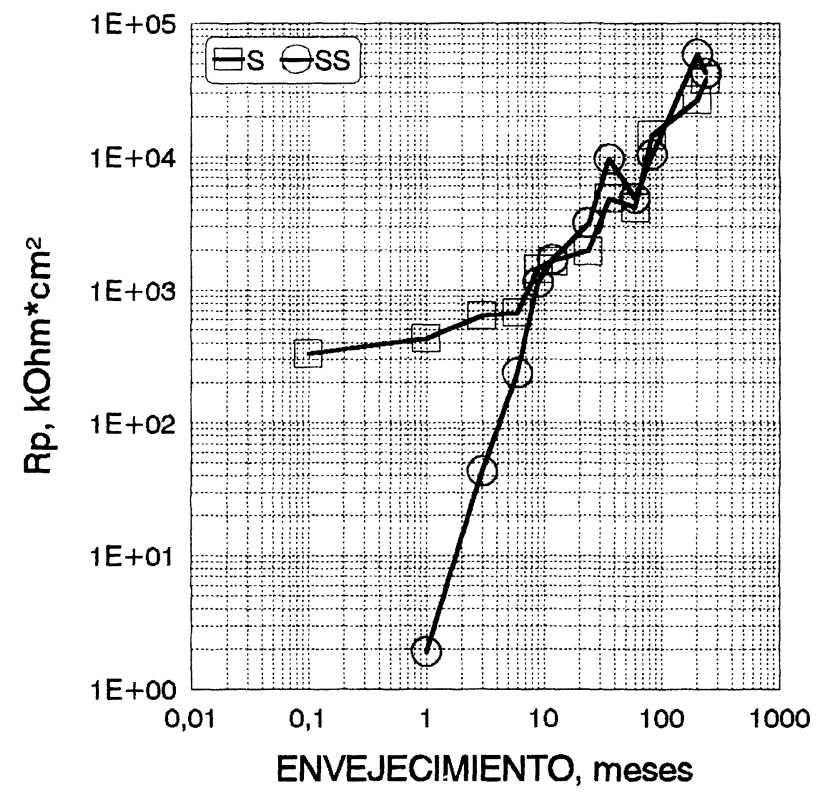

FIG. 8.- Cambios de la resistencia de la capa porosa, deducidos de los diagramas de impedancia, con el tiempo de exposición a la atmósfera de Madrid, de probetas sin sellar y selladas $45 \mathrm{~min}$ en agua desionizada a ebullición.

FIG. 8.- Changes in the porous layer resistance estimated from the impedance diagrams-with the exposure time in the atmosphere of Madrid, corresponding to unsealed specimens and sealed for 45 min in boiling water specimens.

\section{CONCLUSIÓN}

Se reduce sustancialmente, mediante la utilización de aditivos orgánicos adecuados, el tiempo del sellado hidrotérmico tradicional, o la temperatura del mismo.

\section{Agradecimiento}

Este trabajo ha sido financiado por la Comisión Interministerial de Ciencia y Tecnología (CICYT) (Proyecto MAT95-0060).

\section{REFERENCIAS}

(1) BRACE, A.W. The technology of anodizing aluminium, Tecnicopy Ltd., Stonenhouse, Gloucestershire (R.U): 119.

(2) Kalantary, M.R., Gabe, D.R. y Ross, D.H. Plat. Surf. Finish. 80, 1993: 52 - 56.

(3) Wernick, S., Pinner, R. y Sheasby, P. The surface treatments of aluminium and its alloys. $5^{\text {a }}$ Ed. ASM International and Finishing Publ. Ltd. Metals Park, (Ohio, EE.UU,) 1987: 801-806.

(4) Lizarbe, R. y de La Orden, M.A. Rev. Metal. Madrid. 20 (1) 1984: 44-51.

(5) Lizarbe, R. Rev. Metal. Madrid. 22 (4) 1986: 235-242.

(6) Kalantary, M.R., Gabe, D.R. y Ross, D.H. J. Appl. Electrochem. 22, 1992: 268-276.

(7) Lizarbe, R., Gonzalez, J.A., Otero, E. y Lopez, V. Aluminium. 69, 1993: 548.

(8) Gonzalez, J.A., Lopez, V., Otero, E., Bautista, A., Lizarbe, R., Barba, C. y Baldonedo, J.L. Corros. Sci. 39, 1997: 1.109 .

(9) LopeZ, V. y col. (Pendiente de publicación).

(10) Normas UNE 38016, 3817 y 38018, o ISO 2143 y 2931. 